Open Access

\title{
Elevated IL-33 promotes expression of MMP2 and MMP9 via activating STAT3 in alveolar macrophages during LPS-induced acute lung injury
}

Yafeng Liang ${ }^{1,2}$, Nengli Yang ${ }^{3}$, Guoquan Pan², Bingxin Jin², Shufen Wang ${ }^{2}$ and Wei $\mathrm{Ji}^{{ }^{*}}$

* Correspondence: szdxjiwei@163.com

${ }^{1}$ Department of Pediatric

Pulmonology, Children's Hospital of Soochow University, No 303, Jingde Road, Suzhou 215003, China

Full list of author information is available at the end of the article

\section{Abstract}

Background: Pulmonary inflammation and endothelial barrier permeability increase in acute lung injury (ALI) and acute respiratory distress syndrome (ARDS) induced by pro-inflammatory cytokines and matrix metalloproteinases (MMPs). However, the relationship between pro-inflammatory cytokines and MMPs in ALI/ARDS remains poorly understood.

Methods: A lipopolysaccharide (LPS)-induced ALI rat model was established through intratracheal instillation. The wet/dry ratios of lung tissues were measured, and bronchoalveolar lavage fluid (BALF) was collected to test protein concentrations, total cell/macrophage numbers, and pro-inflammatory cytokine levels. LPS-treated alveolar macrophages were utilized in in vitro experiments. The expression and secretion of MMPs were respectively detected using quantitative PCR, Western blotting and ELISA assays.

Results: The levels of IL-33 and MMP2/9 in BALF increased in all the ALI rats with severe lung injury. LPS-induced IL-33 autocrine upregulated the expression of MMP2 and MMP9 through activating STAT3. Neutralizing IL-33 in culture medium with specific antibodies suppressed the expression and secretion of MMP2 and MMP9 in LPS-treated alveolar macrophages. Consistently, eliminating IL-33 decreased the levels of MMP2 and MMP9 in BALF and alleviated lung injury in ALI rats.

Conclusion: The IL-33/STAT3/MMP2/9 regulatory pathway is activated in alveolar macrophages during acute lung injury, which may exacerbate the pulmonary inflammation.

Keywords: Acute lung injury (ALI), IL-33, MMP2, MMP9, STAT3

\section{Background}

Acute lung injury(ALI), also known as acute respiratory distress syndrome (ARDS), is characterized by a serious inflammatory reaction in the lung, and leads to serious hypoxaemia and poor pulmonary compliance in both children and adults [1, 2]. Disruption of the alveolar-capillary unit, breakdown of the barrier, and gas exchange are clinical manifestations of ALI/ARDS [3]. Although new therapies, such as extracorporeal membrane oxygenation and lung protective ventilation strategy, have been applied, the

(c) The Author(s). 2018 Open Access This article is distributed under the terms of the Creative Commons Attribution 4.0 International License (http://creativecommons.org/licenses/by/4.0/), which permits unrestricted use, distribution, and reproduction in any medium, provided you give appropriate credit to the original author(s) and the source, provide a link to the Creative Commons license, and indicate if changes were made. The Creative Commons Public Domain Dedication waiver (http://creativecommons.org/ publicdomain/zero/1.0/) applies to the data made available in this article, unless otherwise stated. 
mortality rate of ALI patients is still as high as $20-50 \%$ [4]. Thus, it is urgent to develop more effective treatments for ALI.

The uncontrolled inflammatory response is a key step during the development of ALI/ARDS. Pro-inflammatory immune cells and the cytokines released by them can increase the permeability of the alveolar-capillary barrier, leading to pulmonary dysfunction [5]. Alveolar macrophages (AMs) are immune cells accounting for 95\% of airspace leukocytes [6]. An essential process in ALI, activation of AMs leads to the oversecretion of various inflammatory cytokines, such as TNF- $\alpha$, IFN- $\gamma$, and interleukins [7]. As a newly identified member of the interleukin (IL)-1 family, IL-33 can aggravate inflammatory reactions and increase microvascular permeability, two processes that decrease the survival rate in LPS-induced ALI mice [8]. In our previous study, the plasma level of IL-33 was found to be higher in ALI patients than in the healthy [9]. This evidence suggests a potential association between IL-33 and ALI.

Matrix metalloproteinases (MMPs) are involved in ALI. MMPs-mediated degradation of proteins in the alveolar epithelial-endothelial unit, including intercellular junction proteins, basement membrane (BM) and proteins anchoring cells to the $\mathrm{BM}$, is a central process in ALI [10]. Among these MMPs, MMP2 and MMP9 are being intensely studied, and their levels are elevated in BALF from ALI patients [11]. The in vivo lung injury model demonstrated a rise of both MMP-2 and -9 in BALF that correlated with the alveolar-arterial oxygen gradient $\left(\mathrm{PA}-\mathrm{a}, \mathrm{O}_{2}\right)$ [12]. Also, in an immune complex deposition model, mice deficient in MMP-9 developed less severe lung injury than wild type mice [13]. However, the mechanisms underlying the rise of MMP2/9 during ALI remain elusive.

Here, we investigated the association between IL-33 and MMP2/9 in an LPS-induced ALI model in vitro and in vivo. Oversecretion of IL-33 promoted the expression of MMP2/9 by activating STAT3 in LPS-treated alveolar macrophages. The regulatory mechanism was further researched with LPS-induced ALI rats.

\section{Materials and methods}

LPS-induced ALI rat model

All 6-week old male Wistar rats were obtained from Wenzhou Medical University and maintained in a pathogen-free environment. Nine rats were assigned to each treatment group and a control group. The ALI model was established following the well-established protocol. In brief, the rats were anesthetized with 3\% sodium pentobarbital, followed by the instillation of $2 \mathrm{mg} / \mathrm{kg}$ LPS (Sigma-Aldrich, St. Louis, MO, USA) solution into the tracheas. Instead of LPS solution, an equal volume of normal saline was used in the control group. For neutralizing IL-33, the rats received an intratracheal instillation of anti-IL-33 antibodies (5 $\mu \mathrm{g}$, ab187060, Abcam, Cambridge, UK) or isotype IgG (ab172730, Abcam) $1 \mathrm{~h}$ after the LPS treatment. Then the rats were allowed to recover and were euthanized $24 \mathrm{~h}$ later with pentobarbital. All animal procedures were approved by the Ethics Committee of the Second Affiliated Hospital of Wenzhou Medical University.

\section{Lung wet/dry ratio analysis}

After the rats were euthanized, their lungs were harvested and weighed immediately. Then the blood on the lung surface was washed away, and the lungs were dried at $60^{\circ}$ 
$\mathrm{C}$ for $72 \mathrm{~h}$. The dried specimens were weighed again, and the wet/dry ratio was calculated accordingly.

\section{BALF collection and inflammatory cell analysis}

The BALF was collected at $24 \mathrm{~h}$ after LPS treatment. Using a tracheal cannula, the lung was washed with $2 \mathrm{ml}$ of normal saline three times. All flushing fluid was collected. The lavaged sample was then centrifuged at $1500 \times \mathrm{g}$ for $10 \mathrm{~min}$ at $4{ }^{\circ} \mathrm{C}$ and the supernatant was collected for protein concentration analysis. Total protein concentration in the supernatant was measured. Each cell pellet was re-suspended in PBS and the total cell number determined in an automatic blood cell analyzer (Sysmex). Macrophages were marked with F4/80 antibodies or FITC-secondary antibodies and then analyzed and sorted with flow cytometry.

\section{Enzyme-linked immunosorbent assay (ELISA)}

Concentrations of IL-33 (BMS2048), TNF- $\alpha$ (KRC3011), MMP2 (KHC3081), MMP9 (BMS2016-2), TIMP1 (ERTIMP1), IL-6 (BMS625), IL-10 (BMS629), and IFN- $\gamma$ (BMS621) in BALF or cell culture medium were determined using their specific enzyme-linked immunosorbent assay (ELISA) kits (Thermo Fisher Scientific) according to the manufacturer's instructions.

\section{Cell cultures and treatment}

NR8383 AMs (Cell Bank of the Chinese Academy of Sciences, Shanghai, China) were derived from Sprague Dawley rats and cultured with Ham's F-12 K medium containing 15\% FBS (Gibco). $1 \times 10^{6}$ NR8383 cells were stimulated with $1 \mu \mathrm{g} / \mathrm{mL}$ LPS (Sigma-Aldrich) or 100, 200, $400 \mathrm{pg} / \mathrm{mL}$ recombinant IL-33 (Novoprotein, Shanghai, China). For neutralizing IL-33, IL-33 antibody was added to the culture medium of NR8383 cells $1 \mathrm{~h}$ after the LPS treatment.

\section{Western blotting}

The whole cell protein was obtained with cold cell lysis buffer and the total protein concentration was measured using the Bradford protein assay (Bio-Rad, Hercules, CA, USA). Equal amounts of protein were separated on 8-12\% SDS-PAGE gel and transferred to a nitrocellulose membrane. The membrane was blocked with 5\% milk and then incubated with primary antibodies (MMP2 (ab92536), MMP9 (ab38898), ST2 (ab228543), IL-1RAP (ab8110), and p65 (ab16502), Abcam, 1:1000; p-STAT3 (Y705, \#4113), STAT3 (\#12640), p-MAPK (\#4511), and MAPK (\#9212), Cell Signaling Technology, Beverly, MA, USA, 1:1000; H3 (sc-517576) and GAPDH(sc-32233), Santa Cruz, Dallas, TX, USA, 1:2000) at $4{ }^{\circ} \mathrm{C}$ overnight. Next, the membranes were incubated with appropriate secondary antibodies at room temperature for $1 \mathrm{~h}$. IRDye $800 \mathrm{CW}$ - or IRDye 680-conjugated secondary antibodies (1:10000) were used for staining and then the proteins were detected using an Odyssey infrared imaging system (both from LI-COR, Lincoln, NE, USA).

\section{Quantitative real-time PCR}

Extraction of total RNA from NR8383 cells was performed with RNAiso Plus reagent and further reverse-transcribed using a PrimeScript RT reagent kit (both from Takara, Tokyo, 
Japan). SYBR-Green mix (Roche) was used to carry out quantitative PCR according to the manufacturer's instructions. Target gene expression was normalized to $\beta$-actin levels in respective samples as an internal control and calculated using the $2^{-\Delta \Delta \mathrm{Cq}}$ method, and the relative mRNA expression was further calculated through normalizing to the control group.

\section{Statistical analysis}

The software SPSS 13.0 and GraphPad Prism 5 were used in the statistical analyses. Group distributions were performed with Student's t-test or analysis of variance (ANOVA). $P<0.05$ was considered statistically significant.

\section{Results}

\section{Establishment of acute lung injury (ALI) model}

In order to establish the in vivo ALI model, LPS was used to induce ALI in the rats. The lung tissues of ALI rats were collected at $24 \mathrm{~h}$ after treatment with or without LPS. The results showed that LPS caused severe pulmonary edema, as indicated by an increased lung wet/dry ratio (Fig. 1a). This observation was further confirmed by the increase of protein concentration in BALF from LPS-treated rats (Fig. 1b). To investigate the level of pro-inflammatory cells and cytokines in ALI, infiltration of total cells and macrophages was evaluated after LPS treatment. The total cells and macrophages were evidently enriched in BALF from LPS-treated rats (Fig. 1c and d). Additionally, the concentrations of IL-33, TNF- $\alpha$, MMP2, MMP9 and TIMP1 in the BALF were significantly elevated (Fig. 1e-i). Furthermore, we purified the alveolar macrophages (AMs) with positive F4/80 staining from BALF. Consistently, the AMs from LPS-treated rats showed a higher level of IL-33 mRNA than those from the control group (Fig. 1j). Notably, as an IL-33 receptor, the ST-2 level was increased in the AMs from LPS-treated rats but the IL-1RAP level remained unchanged (Fig. 1k). Considering the inflammatory response induced by LPS, the status of the inflammatory signaling, p-STAT3, was also examined in the purified AMs. As shown in Fig. 1l, the level of p-STAT3 was significantly increased in the AMs purified from LPS-treated rats. Altogether, our findings indicated that the acute pulmonary inflammatory response was triggered in LPS-induced ALI rats.

\section{LPS induced expression of ST-2 and secretion of IL-33, TNF-a, MMP2, and MMP9 in pri- mary AMs}

Primary AMs were obtained through purifying the alveolar macrophages (AMs) in BALF from normal rats. LPS induced secretion of IL-33, TNF- $\alpha$, MMP2, and MMP9 in primary AMs (Fig. 2a-d). Meanwhile, ST-2 was upregulated but IL-1RAP showed no change (Fig. 2e). STAT3 was also activated by LPS in the primary AMs (Fig. 2f). Therefore, the results suggest that primary AMs can be activated by LPS.

LPS induced secretion of IL-33, TNF-a, MMP2, MMP9, and TIMP1 in AM cell line NR8383 Considering the critical role of AMs in regulating pro-inflammatory events during ALI, we examined the effect of LPS on the secretion of IL-33, TNF- $\alpha$, MMP2, and MMP9 in the AM cell line NR8383. Levels of TNF- $\alpha$, MMP2, and MMP9 in culture medium increased in a time-dependent manner after LPS treatment, while the IL-33 level peaked at $12 \mathrm{~h}$ after LPS treatment (Fig. 3a-d). Although TIMP1 acts as a natural MMP 
A

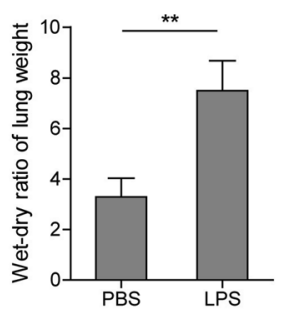

D

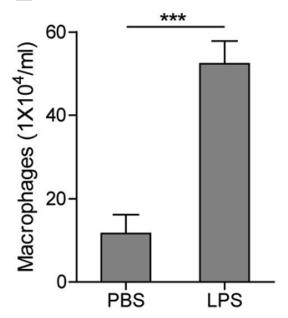

G

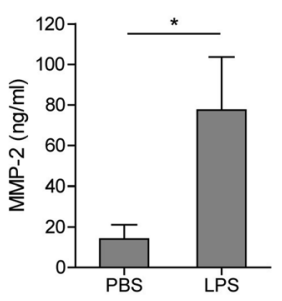

J

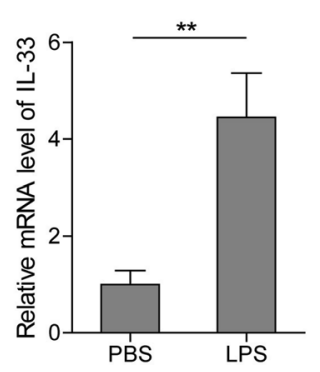

B

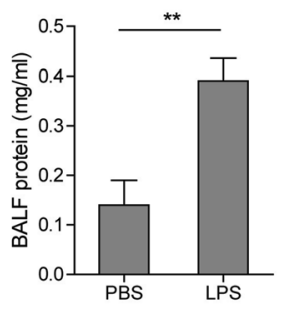

E

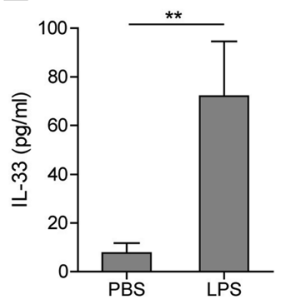

$\mathrm{H}$

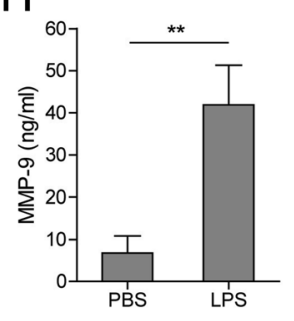

K

Purified F4/80+ AMs

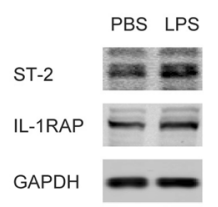

$L$

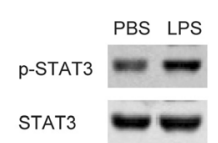

C

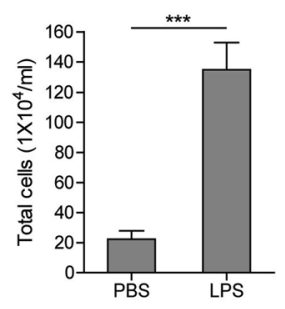

F

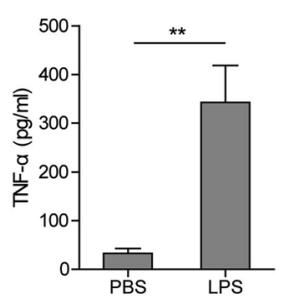

I

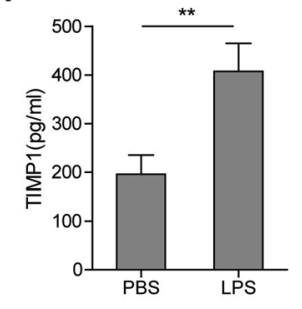

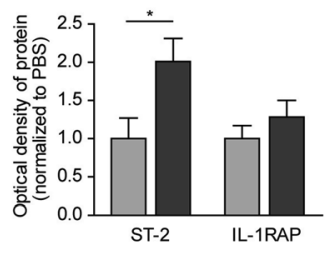

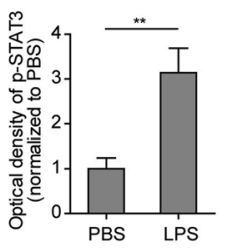

Fig. 1 Establishment of LPS-induced ALI rat model. (a-d) LPS-induced lung injury in experimental rats, as characterized by lung wet/dry ratio (A), protein contents in BALF (b), total cells in BALF (c) and macrophages in BALF (d). (e-i) Levels of IL-33 (e), TNF-a (f), MMP2 (g), MMP9 (h), and TIMP1 (i) in BALF of LPS-induced ALI rats. (j) mRNA level of IL-33 in F4/80+ AMs purified from BALF of LPS-induced ALI rats. (k) Protein levels of ST-2 and IL-1RAP in F4/80+ AMs purified from BALF of LPS-induced ALI rats. (I) Protein levels of p-STAT3 and STAT3 in F4/80+ AMs purified from BALF of LPS-induced ALI rats. Data are shown as means \pm SD ( $N=9$ per group). Two-tailed $t$ test was used in the statistical analyses. ${ }^{*} p<0.05$, ${ }^{* *} p<0.01,{ }^{* * *} p<0.001$ vs. control

inhibitor, the secretion of TIMP1 was also promoted by LPS (Fig. 3e). These results suggest that the levels of IL-33, TNF- $\alpha$, MMP2, MMP9, and TIMP1 in BALF from the LPS-induced ALI rats may be raised by the alveolar macrophages which were activated by LPS. 


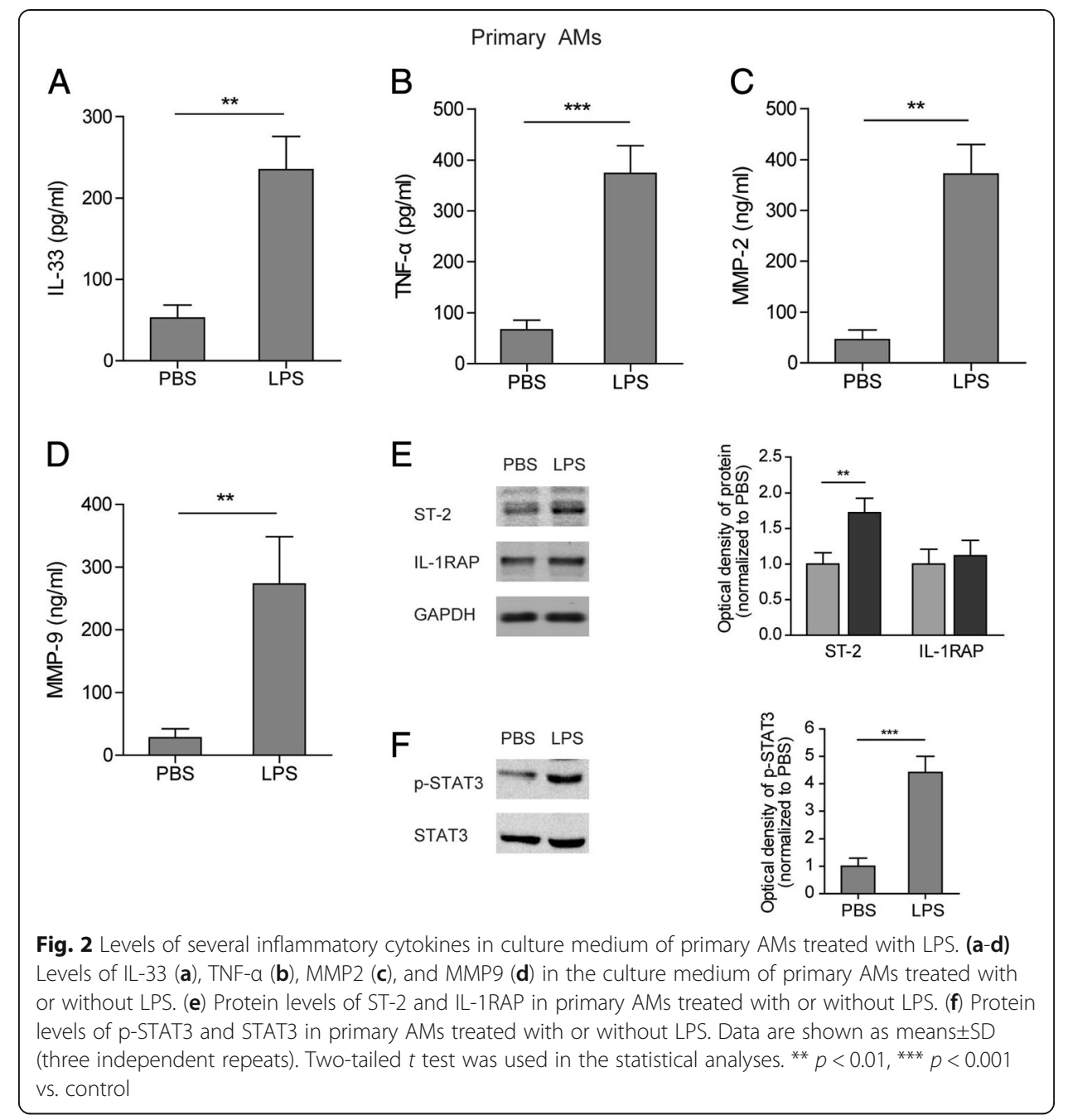

\section{IL-33 increased expression of MMP2 and MMP9 via STAT3 signaling in AM cell line NR8383}

To determine whether the secretion of MMP2 and MMP9 is induced by IL-33, NR8383 cells were stimulated with recombinant IL-33 protein. It was found that the concentrations of MMP2 and MMP9 in culture medium were increased in an IL-33-dose-dependent manner (Fig. 4a and b). Consistently, the mRNA and protein levels of MMP2 and MMP9 were upregulated in NR8383 cells treated with IL-33 (Fig. $4 \mathrm{c}$-e). As a powerful signal transducer, STAT3 is essential for the interleukin-mediated activation of macrophages. Here, we found that IL-33 induced the phosphorylated activation of STAT3 in primary AMs (Fig. 4f). Blocking the activation of STAT3 with the specific inhibitor stattic attenuated the IL-33-induced expression and secretion of MMP2 and MMP9 in NR8383 cells, with no effect on activation of the MAPK or NFkb pathway (Fig. 4g-1). Additionally, we investigated whether other inflammatory cytokines were also produced through IL-33/STAT3 signaling in AMs. The results showed that IL-33 induced the secretion of TNF- $\alpha$, IL-6, IL-10, and IFN- $\gamma$ in NR8383 cells, which was not significantly changed after stattic addition (Fig. 4m). Similarly to stattic treatment, knocking down STAT3 with siRNA significantly decreased the mRNA levels of 

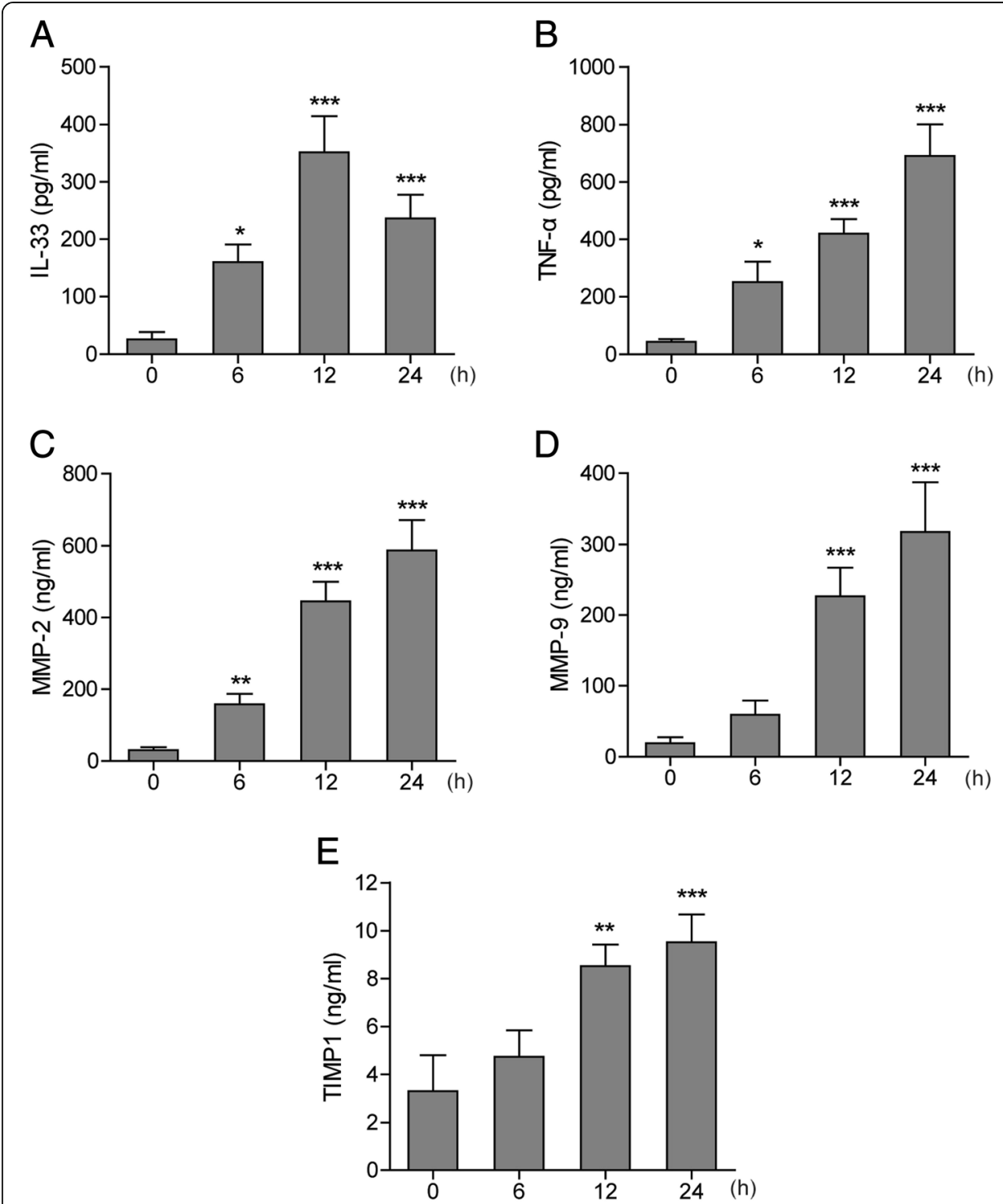

Fig. 3 Levels of several inflammatory cytokines in culture medium of NR8383 cells after LPS stimulation. Levels of IL-33 (a), TNF-a (b), MMP2 (c), MMP9 (d), and TIMP1 (e) in the medium at indicated time points after LPS treatment $(1 \mu \mathrm{g} / \mathrm{mL})$ in NR8383 cells. Data are shown as means \pm SD (three independent repeats). One-way ANOVA was used in the statistical analyses. ${ }^{*} p<0.05,{ }^{* *} p<0.01,{ }^{* * *} p<0.001$ vs. zero time point

MMP2 and MMP9 in NR8383 cells as well as their concentrations in culture medium (Fig. 5a-f). Therefore, these results demonstrate that IL-33 promotes expression of MMP2 and MMP9 in AMs through activating STAT3.

\section{Neutralizing IL-33 inhibited the LPS-induced MMP2/9 expression and lung injury}

To verify the role of IL-33 in LPS-induced ALI, IL-33 specific antibodies were administered into culture medium to neutralize the secreted IL-33 when AMs were being treated with LPS. It showed that IL-33 antibodies significantly lowered the mRNA and protein levels of MMP2 and MMP9 in LPS-treated NR8383 cells, as well as their medium concentrations (Fig. 6a-e). To further evaluate the protective effect of IL-33 


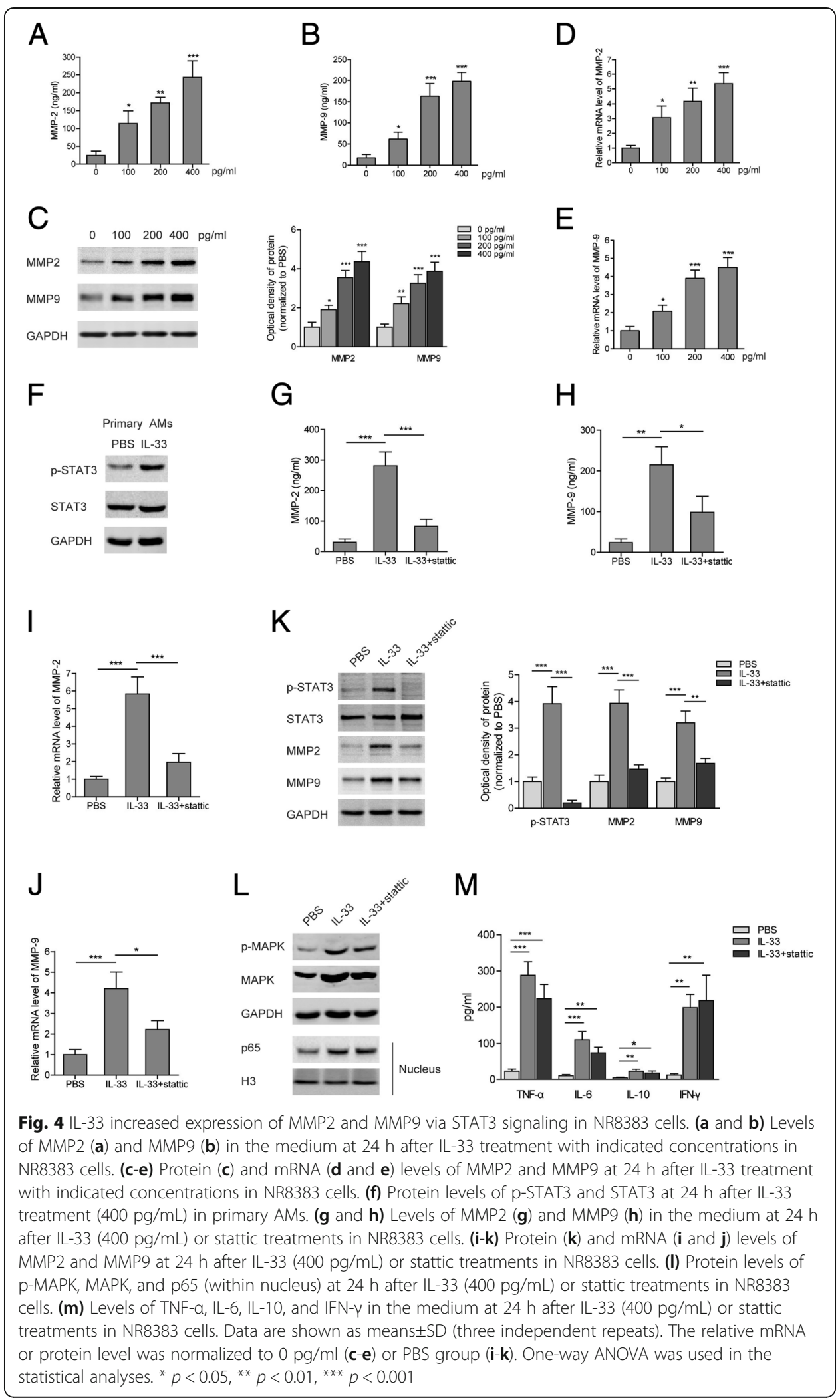




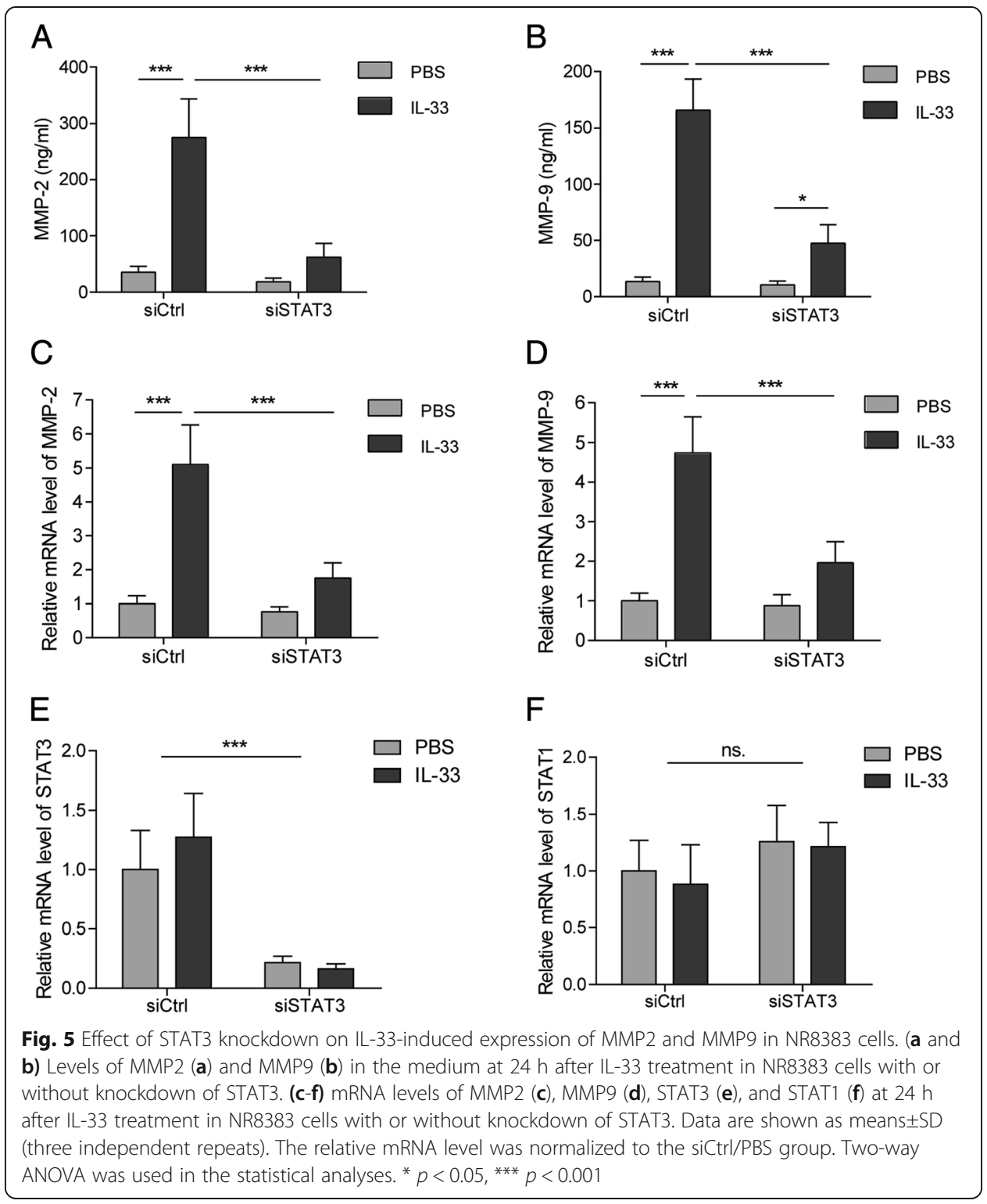

antibody in the LPS-induced ALI model, the rats received an intratracheal instillation of IL-33 antibody after LPS administration to neutralize the secreted IL-33. The results showed that treatment with anti-IL-33 antibodies obviously curbed the edema development and inflammatory cell infiltration in the lung tissues from LPS-induced ALI rats (Fig. 7a-d). Moreover, the concentrations of MMP2 and MMP9 in BALF from the LPS-treated rats were substantially decreased after the addition of IL-33 antibodies (Fig. 7e and f). Taken together, IL-33 displays a central role in LPS-induced lung inflammation/injury and MMP2/9 secretion.

\section{Discussion}

In the present study, an in vivo LPS-induced ALI rat model and an in vitro model using AM NR8383 cells were established to investigate the link between IL-33 and ALI. BALF from the in vivo model showed an increased IL-33 level, which is consistent with the 


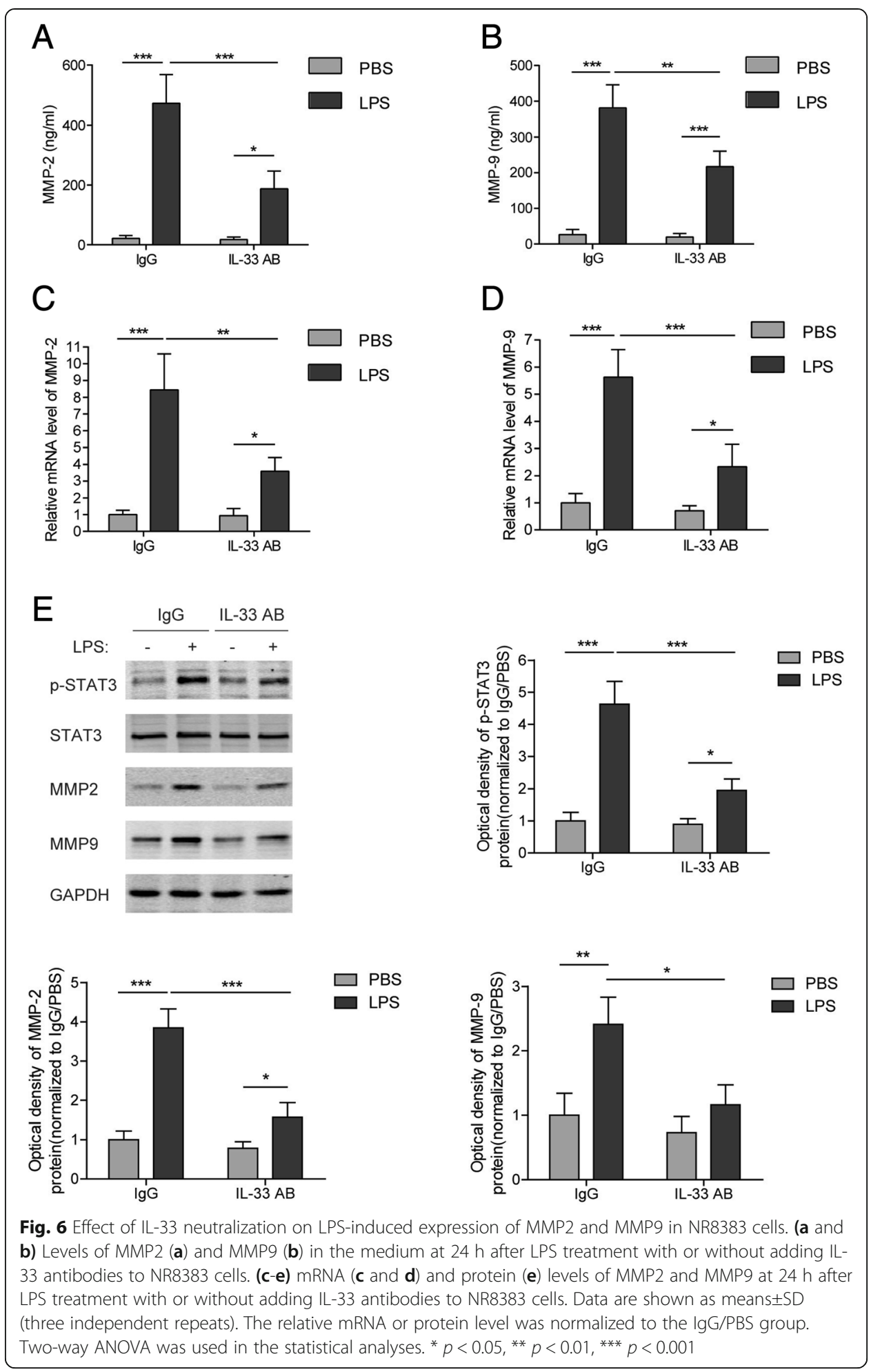

observation in LPS-induced ALI mice [14]. The expression of IL-33 was boosted by LPS in AMs, suggesting the ability of AMs to produce IL-33 during LPS-induced ALI. AMs, once activated by toll-like receptors (TLRs), serve as the first line of defense against invaders into the lung [15]. Wang et al. reported that the NF-kB pathway 


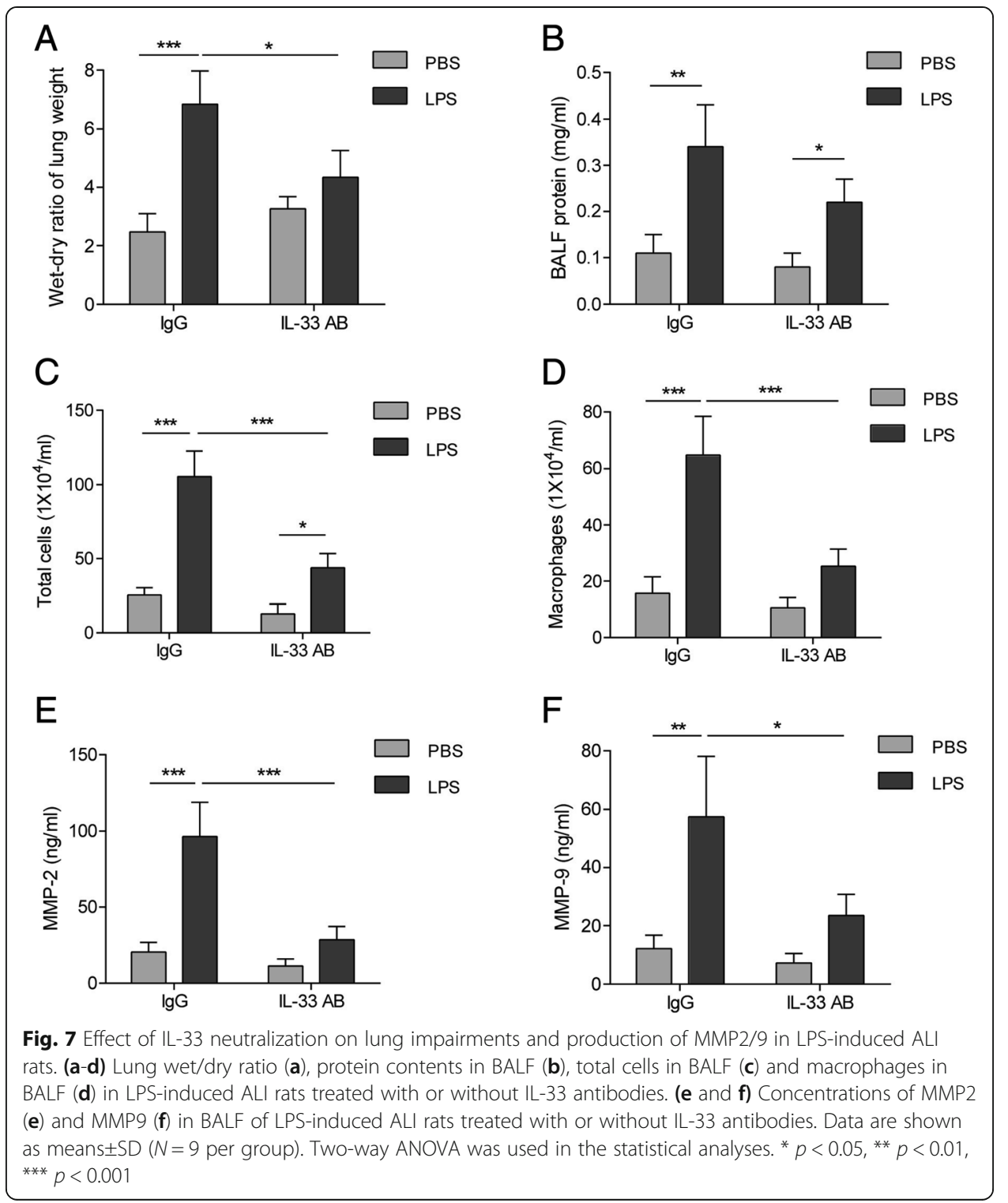

aberrantly activated in BALF-derived macrophages aroused ARDS in rats [16]. Therefore, we supposed that the LPS-induced IL-33 secretion requires activation of the TLR4/NF- $\kappa B$ pathway since NF- $\kappa B$ is the central mediator of inflammation. Several studies have demonstrated that IL-33 directly up-regulated expression of the LPS receptor TLR4 in macrophages, and this up-regulation in turn exaggerated the activation of NF- $\mathrm{kB}$ and increased the production of pro-inflammatory cytokines [17, 18]. Furthermore, Fu et al. found that the up-regulation of IL-33 in LPS-induced ALI mice also involved HMGB1, a leading factor for the cascade amplification of inflammation [19].

Except for IL-33, LPS stimulation immediately activated MMP2 and MMP9 production in BALF from ALI rats, which is also validated by the results of in vitro studies using NR8383 cells. However, the level of IL-33 secreted by NR8383 cells peaked at $12 \mathrm{~h}$ after LPS treatment, while levels of MMP2 and MMP9 continued to increase, more evidently after $12 \mathrm{~h}$. As the time-course study revealed that relatively early expression of IL-33 was followed by increased production of MMP2 and MMP9 after 
LPS challenge in AMs, we hypothesize that autocrine or paracrine IL-33 release after LPS stimulation further activates the downstream inflammatory pathways which up-regulate the expression of MMP2 and MMP9. Neutralizing the secreted IL-33 after LPS treatment not only decreased the production of MMP2 and MMP9 but also protected the rats from LPS-induced ALI, indicating that IL-33 may function as an inflammatory mediator in ALI development.

A few studies have been conducted to investigate the mechanisms behind IL-33-mediated expression of MMP2 and MMP9 in macrophages. Ariyoshi et al. found that the transcription of MMP9 was induced by AP-1 activation, which is dependent on ERK1/2/CREB and NF- $\mathrm{kB}$ cascades in IL-33-stimulated macrophages [20]. However, in the present study, STAT3 was identified as a required mediator in IL-33-induced expression of MMP2 and MMP9. Although STAT3 directly activates the transcription of MMP2 and MMP9 in cancer cells [21, 22], the regulatory mechanisms in alveolar macrophages should be further validated.

In conclusion, IL-33 production increases in LPS-induced ALI rats and LPS-treated alveolar macrophages. Then, the secreted IL-33 induces the expression of MMP2 and MMP9 in a STAT3-dependent manner. Disrupting the IL-33/STAT3/MMP2/9 pathway by neutralizing IL-33 relieves the pulmonary inflammation and injury in LPS-induced ALI rats, suggesting that IL-33 could be a potential therapeutic target for ALI.

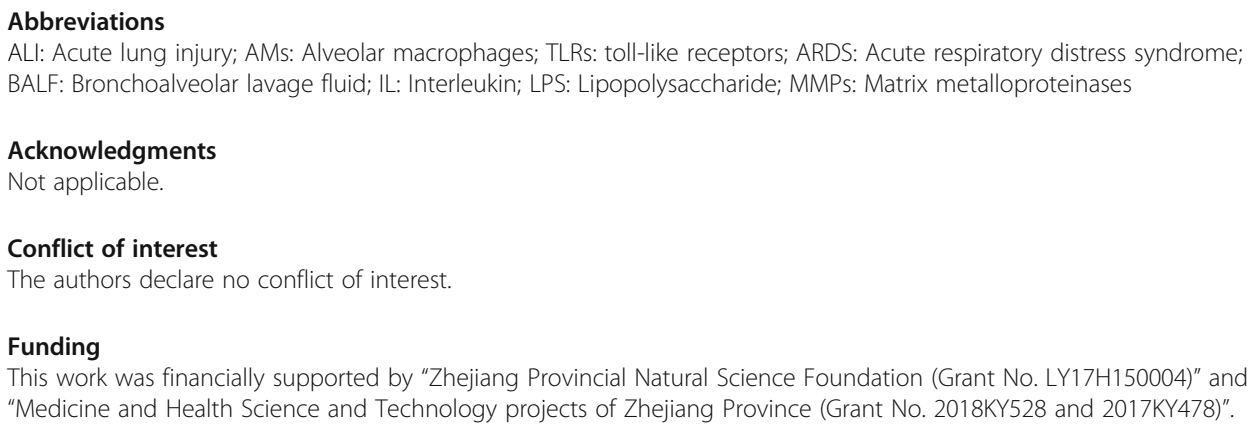

Consent for publication

Not applicable.

\section{Publisher's Note}

Springer Nature remains neutral with regard to jurisdictional claims in published maps and institutional affiliations.

\section{Author details}

'Department of Pediatric Pulmonology, Children's Hospital of Soochow University, No 303, Jingde Road, Suzhou 215003, China. 'Department of Pediatric Intensive Care Unit, The Second Affiliated Hospital \& Yuying Children's Hospital, Wenzhou Medical University, Wenzhou 325000, China. ${ }^{3}$ Department of Anesthesiology, The First Affiliated Hospital of Wenzhou Medical University, Wenzhou 325000, China. 
Received: 20 June 2018 Accepted: 12 October 2018

Published online: 31 October 2018

\section{References}

1. Umbrello M, Formenti P, Bolgiaghi L, Chiumello D. Current concepts of ARDS: a narrative review. Int J Mol Sci. 2016;18(1).

2. Confalonieri M, Salton F, Fabiano F. Acute respiratory distress syndrome. European respiratory review : an official journal of the European Respiratory Society. 2017;26(144).

3. Fanelli V, Ranieri VM. Mechanisms and clinical consequences of acute lung injury. Annals of the American Thoracic Society. 2015;12(Suppl 1):S3-8.

4. Villar J, Blanco J, Kacmarek RM. Current incidence and outcome of the acute respiratory distress syndrome. Curr Opin Crit Care. 2016;22(1):1-6.

5. Matthay MA, Zimmerman GA. Acute lung injury and the acute respiratory distress syndrome: four decades of inquiry into pathogenesis and rational management. Am J Respir Cell Mol Biol. 2005;33(4):319-27.

6. Martin WJ 2nd, Wu M, Pasula R. A novel approach to restore lung immunity during systemic immunosuppression. Trans Am Clin Climatol Assoc. 2005;116:221-6 discussion 6-7.

7. Aggarwal NR, King LS, D'Alessio FR. Diverse macrophage populations mediate acute lung inflammation and resolution. American journal of physiology Lung cellular and molecular physiology. 2014;306(8):L709-25.

8. Zhang $Y, L v R$, Hu X, Jiang L, Xiao D, Sun Y, et al. The role of IL-33 on LPS-induced acute lung injury in mice. Inflammation. 2017;40(1):285-94.

9. Pan G, Liang Y, Chen X, Lu L, Wang L, Wang M, et al. Pro-inflammatory and pro-apoptotic role of IL-33 in the human acute lung injury (ALI) development. Current Signal Transduction Therapy. 2015;10(1):48-55.

10. Davey A, McAuley DF, O'Kane CM. Matrix metalloproteinases in acute lung injury: mediators of injury and drivers of repair. Eur Respir J. 2011;38(4):959-70.

11. O'Kane CM, McKeown SW, Perkins GD, Bassford CR, Gao F, Thickett DR, et al. Salbutamol up-regulates matrix metalloproteinase-9 in the alveolar space in the acute respiratory distress syndrome. Crit Care Med. 2009;37(7):2242-9.

12. Eichler W, Bechtel JF, Schumacher J, Wermelt JA, Klotz KF, Bartels C. A rise of MMP-2 and MMP-9 in bronchoalveolar lavage fluid is associated with acute lung injury after cardiopulmonary bypass in a swine model. Perfusion. 2003;18(2):107-13.

13. Warner RL, Lewis CS, Beltran L, Younkin EM, Varani J, Johnson KJ. The role of metalloelastase in immune complexinduced acute lung injury. Am J Pathol. 2001;158(6):2139-44.

14. Yin H, Li X, Yuan B, Zhang B, Hu S, Gu H, et al. Heme oxygenase-1 ameliorates LPS-induced acute lung injury correlated with downregulation of interleukin-33. Int Immunopharmacol. 2011;11(12):2112-7.

15. Schwartz MD, Moore EE, Moore FA, Shenkar R, Moine P, Haenel JB, et al. Nuclear factor-kappa B is activated in alveolar macrophages from patients with acute respiratory distress syndrome. Crit Care Med. 1996;24(8):1285-92.

16. Wang $S, L i Z$, Chen Q, Wang L, Zheng J, Lin Z, et al. NF-kappaB-induced MicroRNA-211 inhibits Interleukin-10 in macrophages of rats with lipopolysaccharide-induced acute respiratory distress syndrome. Cell Physiol Biochem. 2018; 45(1):332-42.

17. Espinassous Q, Garcia-de-Paco E, Garcia-Verdugo I, Synguelakis M, von Aulock S, Sallenave JM, et al. IL-33 enhances lipopolysaccharide-induced inflammatory cytokine production from mouse macrophages by regulating lipopolysaccharide receptor complex. J Immunol. 2009;183(2):1446-55.

18. Ohno T, Oboki K, Morita H, Kajiwara N, Arae K, Tanaka S, et al. Paracrine IL-33 stimulation enhances lipopolysaccharidemediated macrophage activation. PLoS One. 2011;6(4):e18404.

19. Fu J, Lin SH, Wang CJ, Li SY, Feng XY, Liu Q, et al. HMGB1 regulates IL-33 expression in acute respiratory distress syndrome. Int Immunopharmacol. 2016;38:267-74.

20. Ariyoshi W, Okinaga T, Chaweewannakorn W, Akifusa S, Nisihara T. Mechanisms involved in enhancement of matrix metalloproteinase-9 expression in macrophages by interleukin-33. J Cell Physiol. 2017;232(12):3481-95.

21. Xuan X, Li S, Lou X, Zheng X, Li Y, Wang F, et al. Stat3 promotes invasion of esophageal squamous cell carcinoma through up-regulation of MMP2. Mol Biol Rep. 2015;42(5):907-15.

22. Feng J, YU SY, Li CZ, Li ZY, Zhang YZ. Integrative proteomics and transcriptomics revealed that activation of the IL-6R/ JAK2/STAT3/MMP9 signaling pathway is correlated with invasion of pituitary null cell adenomas. Mol Cell Endocrinol. 2016:436:195-203.

Ready to submit your research? Choose BMC and benefit from:
- fast, convenient online submission
- thorough peer review by experienced researchers in your field
- rapid publication on acceptance
- support for research data, including large and complex data types
- gold Open Access which fosters wider collaboration and increased citations
- maximum visibility for your research: over 100M website views per year
At BMC, research is always in progress.
Learn more biomedcentral.com/submissions

